\title{
Treatment of Yunnan Baiyao plus Kangfuxin Solution Reduces Inflammatory Response and Prevents Patients with Nasopharyngeal Carcinoma against Radiation-Induced Oral Mucositis
}

\author{
Xiuyu Tang, Jiahui Sun, Jie Deng, and Bin Shi $\mathbb{C}$ \\ The State Key Laboratory Breeding Base of Basic Science of Stomatology (Hubei-MOST) and Key Laboratory for Oral Biomedical \\ Ministry of Education, School and Hospital of Stomatology, Wuhan University, Wuhan, China \\ Correspondence should be addressed to Bin Shi; shibin_dentist@whu.edu.cn
}

Received 25 March 2021; Accepted 27 April 2021; Published 17 May 2021

Academic Editor: Songwen Tan

Copyright (c) 2021 Xiuyu Tang et al. This is an open access article distributed under the Creative Commons Attribution License, which permits unrestricted use, distribution, and reproduction in any medium, provided the original work is properly cited.

\begin{abstract}
Oral mucositis refers to secondary mucosal damage, which usually occurs during cancer treatment. Generally, patients with head and neck cancers receiving radiotherapy will develop mucositis. Oral mucositis usually begins with mucosal inflammation and is characterized by erythema and confluent ulcers. The purpose of the study is to explore the therapeutic effects of Yunnan Baiyao combined with Kangfuxin solution on radiation-induced oral mucositis and the influence on production of inflammatory factors in patients with nasopharyngeal carcinoma (NPC) after radiotherapy. Clinical variables of 90 NPC patients were retrospectively analyzed. All patients underwent combined treatment (normal saline and inhalation of dexamethasone, gentamicin, and vitamin $\mathrm{B}_{12}$ ) $1^{\text {st }}$ after radiotherapy, among which 45 patients received additional treatment of Yunnan Baiyao plus Kangfuxin solution and assigned as the study group. We found that additional treatment of Yunnan Baiyao plus Kangfuxin solution remarkably attenuated pain and dry mouth and reduced the degree of mucosal hyperaemia, edema, and ulceration in NPC patients undergoing radiotherapy $(P<0.05)$. It was also found that additional treatment of Yunnan Baiyao plus Kangfuxin solution notably inhibited the release of inflammatory factors and cancer-related markers, as evidenced by lower serum levels of C-reactive protein (CRP), tumor necrosis factor- $\alpha$ (TNF- $\alpha$ ), interleukin-1 (IL-1), matrix metalloproteinase-9 (MMP-9), serum hypoxia-inducible factor-1 $\alpha$ (HIF-1 $\alpha$ ), and vascular endothelial growth factor (VEGF) detected in the study group than the control group $(P<0.05)$. Additionally, the numbers of $\mathrm{CD}^{+3}$ and $\mathrm{CD}^{+4}$ subpopulations of $\mathrm{T}$ lymphocytes and the ratio of $\mathrm{CD}^{+4} / \mathrm{CD}^{+8}$ in the study group were significantly higher than those in the control group $(P<0.05)$, and the number of $\mathrm{CD}^{+8}$ subpopulations in the study group was significantly lower than those in the control group $(P<0.05)$. In conclusion, these results indicated that additional treatment of Yunnan Baiyao plus Kangfuxin solution reduces inflammatory response and prevents patients with NPC against radiation-induced oral mucositis.
\end{abstract}

\section{Introduction}

Nasopharyngeal carcinoma (NPC) is a common head and neck cancer in Southeast Asia and South China, which has distinct ethnic and regional distribution characteristics [1]. At present, most patients are diagnosed with local advanced or late stage, about $90 \%$ of cases are diagnosed as lymph node metastasis, and about $5-10 \%$ of patients have distant metastasis [2]. NPC has unique clinical biological profiles such as associated Epstein-Barr virus infection and high radiosensi- tivity. Radiotherapy has long been recognized as the mainstay for the treatment of NPC [3]. Although intensitymodulated radiotherapy combined with radiotherapy can control primary NPC, local recurrence and distant metastasis are still the main obstacles to the success of NPC treatment [4]. Additionally, radiation can not only kill malignant tumor cells but also damage normal cells in radiation field and its surrounding area. Oral mucosa is stratified squamous epithelium with rapid renewal and high radiosensitivity. Oral mucositis is a common adverse reaction of NPC after 
radiotherapy [5]. Radiotherapy-induced oral mucositis are characterized by dry mouth, taste dysfunction, oral mucosal congestion, edema, erosion, and ulcer, which seriously affect the patient's eating function, eventually lead to malnutrition and electrolyte disorder [6]. Currently, there are effective mucoprotective strategies for the management of oral mucositis. Severe mucositis can not only affect a patients' quality of life but also result in requirements for narcotic analgesics, total parenteral nutrition, interruption of cancer therapy, prolong hospitalization, and increases of the risk of local and systemic infection [7]. Accumulating evidence has suggested that the pathogenesis of oral mucositis is associated with a cascade of inflammatory events, which chronologically consist of five continuous overlapping phases: initiation, upregulation of inflammation, signaling and amplification, ulceration, and finally, wound healing [8,9]. Oral mucositis caused by radiotherapy and chemotherapy is easy to diagnose but difficult to cure, and in recent years, a variety of medicines have been applied to the prevention and treatment of oral mucositis, but medicines with definite clinical recommended efficacy need to be developed or discovered [10]. Yunnan Baiyao (YNBY), as traditional Chinese medicine, has been attached great importance for hundreds of years, which has significant clinical efficacy in the aspects of hemostasis, improving blood circulation and dispersing blood stasis and anti-inflammation [11, 12]. Kangfuxin solution was extracted from cockroaches, which was defined as Chinese herbal medicine, and studies have indicated that Kangfuxin solution can maintain normal cell function by inhibiting the opening of calcium-dependent potassium channels caused by radiation injury, so as to reduce the pain and discomfort of patients after radiotherapy and chemotherapy [13]. The present study investigates preventive and therapeutic effects of Yunnan Baiyao combined with Kangfuxin solution on radiation-induced oral mucositis and the influence on serum levels of inflammatory factors in patients with NPC after radiotherapy.

\section{Material and Method}

2.1. Patient Selection and Distribution. A total of 90 patients with NPC treated with initial radiotherapy in our hospital were retrospectively analyzed form January 2013 to December 2016. They were divided into study group and control group with 45 cases, respectively, and there were 26 males and 19 females in study group who diagnosed pathologically as squamous cell carcinoma, the age ranged from 34 to 67 years old, with an average age of $48.7 \pm 11.0$ )years. The Karnofsky Performance Scale (KPS) score was 65.9 \pm 4.0 before radiotherapy, and the prescribed radiation dose was $72.0 \pm 2.0 \mathrm{~Gy}$. There were 23 males and 23 females in the control group which diagnosed pathologically as squamous cell carcinoma, the age ranged from 37 to 69 years old, with an average age of $49.4 \pm 10.5$ years. The KPS score was $66.3 \pm 4.5$ before radiotherapy, and the prescribed radiation dose was $72.0 \pm 2.0 \mathrm{~Gy}$. There was no significant difference in age, gender, pathological type, KPS score, and radiation dose between the two groups $(P>0.05)$. The eligible study subjects should meet the following inclusion cri- teria: (a) diagnosed with NPC by pathological examination; (b) aged from 18 to 69 years old; (c) initially diagnosed as NPC and pathologically defined as squamous cell carcinoma; (d) be treated with 30 times of radiotherapy with 70-74 Gy radiation dose each time; (e) the KPS score > 70 before radiotherapy; (f) occurrence of grade I oral mucosal during radiotherapy; $(\mathrm{g})$ the hemoglobin $\geq 100 \mathrm{~g} / \mathrm{L}$, platelet $\geq 75 \times 10^{9} / \mathrm{L}$, white blood cell count $\geq 3.0 \times 10^{9} / \mathrm{L}$, absolute neutrophil count $\geq 1.5 \times 10^{9} / \mathrm{L}$; (h) informed consent was signed. The patients were consistent with any of the below criteria should be excluded: (a) grade II or more oral mucosal reaction occurred during radiotherapy; (b) medical history of radiotherapy to the face and neck; (c) radiotherapy and chemotherapy history of other tumors; (d) complicated by other oral diseases before radiotherapy; (e) immune deficiency; (f) allergic reaction to the drugs in this study; (g) negative treatment compliance; (h) special populations during pregnancy and lactation. Figure 1 shows the screening process of eligible study subjects. The study was approved by the Medical Ethics Committee of our hospital.

2.2. Treatment Procedures. All patients were fixed under the simulator and treated with intensity-modulated radiotherapy (IMRT) with Siemens high-energy linear accelerator. The radiation dose ranged from $60 \mathrm{~Gy}$ to $70 \mathrm{~Gy}$, and the single dose was 2 Gy with 5 days a week. At the first day of radiotherapy, local application of physiological saline combined with dexamethasone, gentamicin, and vitamin $B_{12}$ was used to the control group: dexamethasone $5 \mathrm{mg}$, gentamicin $160,000 \mathrm{u}$, and vitamin B $12 \mathrm{mg}$ were added into $100 \mathrm{~mL}$ of physiological saline as atomization inhalation for 15 minutes each time and twice a day, while Yunnan Baiyao (Yunnan Baiyao Group Co., Ltd., National Medicine Permission Number: Z53020798, specification: $4 \mathrm{~g}$ ) as oral administration and Kangfuxin solution (Inner Mongolia Dual Fufangtai Pharmaceutical Co., Ltd, National Medicine Permission Number: Z15020805, specification: $30 \mathrm{~mL} \times 4$ ) for gargling and local application of physiological saline combined with dexamethasone, gentamicin, and vitamin $\mathrm{B}_{12}$ for atomization inhalation were applied to the study group. The atomization method was the same as that of the control group, Kangfuxin solution $10 \mathrm{~mL}$ was used to gargle for 2 minutes to make it fully contact with the surface of oral mucosa after cleaning the oral cavity in the morning and evening, and food and water should not be eaten after gargling within 1 hour. Yunnan Baiyao powder was orally administrated, with $0.5 \mathrm{~g}$ once and 3 times a day. Fish and bean products were forbidden during medication. The treatment started from the beginning of radiotherapy to the 7 days after the radiotherapy. There were no adverse reactions in the course of medication in the study group and the control group.

\subsection{Classification of Radiation-Induced Oral Mucositis. The} classification of radiation-induced oral mucositis was made based on radiation therapy oncology group (RTOG) [14], which was detailed in Table 1. In addition, patient outcome was also analyzed by scoring symptoms including pain, mucosal hyperaemia and edema, mucosal ulceration, and 


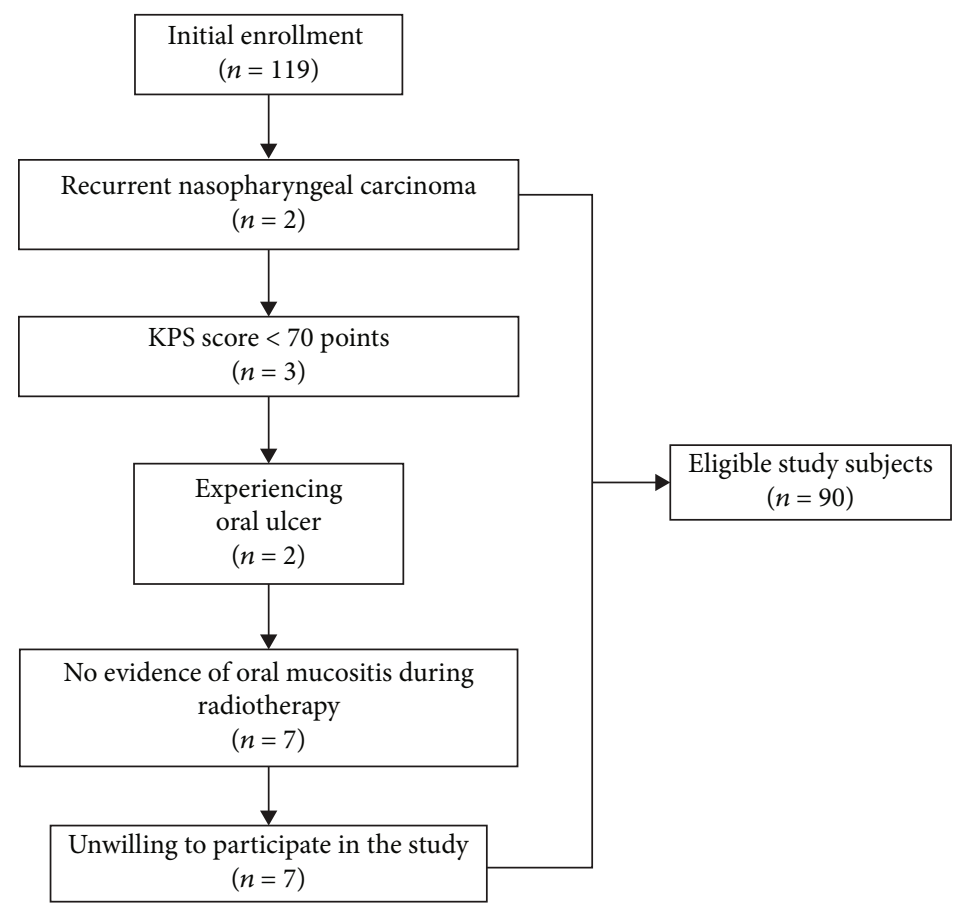

Figure 1: The screening process of eligible study subjects. Finally, 90 eligible patients with nasopharyngeal carcinoma meet predefined inclusion and exclusion criteria and were recruited into the present study.

TABLE 1: Classification of radiation-induced oral mucositis.

\begin{tabular}{|c|c|c|}
\hline Grade & Symptom & Treatment \\
\hline 0 & No evidence of oral mucositis & No requirement \\
\hline 1 & Feeling slight pain but no need of painkillers, with evidence of mild hyperaemia of oral mucosa & No requirement \\
\hline 2 & Feeling moderate pain, visible spotted mucositis, detectable inflammatory substances in the secretion & Administration of painkiller \\
\hline 3 & $\begin{array}{l}\text { Presence of flaky oral mucositis, accounting for } 50 \% \text { of the radiation area, with pain need to be } \\
\text { relieved by anesthetic drugs }\end{array}$ & $\begin{array}{l}\text { Administration of } \\
\text { anesthetic drugs }\end{array}$ \\
\hline 4 & $\begin{array}{c}\text { Mucosal ulceration, bleeding, and erosions occurring in the inflammatory areas, accounting for } \\
\text { more than } 50 \% \text { of radiation area }\end{array}$ & $\begin{array}{l}\text { Treatment discontinued or } \\
\text { changing patterns of nutrition }\end{array}$ \\
\hline
\end{tabular}

dry mouth. The total score, respectively, was 0-6 points for each symptom. The lower the score was, the better the efficacy was.

2.4. Serum Extraction. Two tubes of fasting venous blood $(5 \mathrm{~mL})$ were collected from each patient, and blood collection was performed twice before and after treatment. The blood samples were refrigerated under $4^{\circ} \mathrm{C}$ for 45 minutes and centrifuged with $3500 \mathrm{r} / \mathrm{min}$ for $15 \mathrm{~min}$. The serum was extracted from testing tube, which was immediately stored in a lowtemperature refrigerator (MDF-U5412, SANYO, Japan) at $-80^{\circ} \mathrm{C}$.

2.5. Reagents and Cytokine Determination. Cytokine detection in the serum was achieved by commercial kits (RapidBio, West Hills, CA, USA; C-reactive protein (CRP), tumor necrosis factor- $\alpha$ (TNF- $\alpha$ ), interleukin-1 (IL-1), matrix metalloproteinase-9 (MMP-9), hypoxia-inducible factor-1 $\alpha$ (HIF-1 $\alpha$ ), and vascular endothelial growth factor (VEGF)). Serum cytokine measurement was performed using an enzyme-linked immunosorbent assay (ELISA) method as specified by the kit manufactures at test and reference wavelengths of 450 and $550 \mathrm{~nm}$, respectively. The results were expressed in $\mathrm{mg} / \mathrm{L}, \mu \mathrm{g} / \mathrm{L}$, or $\mathrm{ng} / \mathrm{L}$ and relative to standard curves obtained from titrations of the corresponding recombinant factors provided the kit manufactures. The manufacturers guarantee the specificity of each individual kit.

2.6. Flow Cytometric Analysis of $T$ Cell Subpopulation. Venous blood $(2 \mathrm{~mL})$ was extracted into EDTA anticoagulant tube and centrifuged with lymphocyte separation solution $(2 \mathrm{~mL})$ at the speed of $1500 \mathrm{r} / \mathrm{min}$ for 10 minutes so as to collect the layer containing lymphocyte, which was rinsed with $2 \mathrm{~mL}$ phosphate-buffered solution (PBS), and centrifuged twice at the same speed and time to discard the supernatant. The final cell suspension was divided into two test tubes with $5 \mathrm{~mL}$ volumes, respectively, and have them made with the concentration of $1 \times 106 \mathrm{~mL}^{-1}$. Four test tubes were added with lymphocyte cell suspension $500 \mu \mathrm{L}$, respectively, the monoclonal antibody (McAb) of mouse anti-human $\mathrm{CD}^{+3}$, 
TABLE 2: The scores of symptoms of oral mucositis including pain, mucosal hyperaemia and edema, mucosal ulceration, and dry mouth.

\begin{tabular}{|c|c|c|c|c|c|c|}
\hline Group & Case & Time & Pain & Mucosal hyperaemia and edema & Mucosal ulceration & Dry mouth \\
\hline \multirow{2}{*}{ Control group } & \multirow{2}{*}{45} & Before treatment & $4.20 \pm 1.11$ & $4.37 \pm 1.24$ & $3.97 \pm 0.88$ & $3.73 \pm 0.81$ \\
\hline & & After treatment & $2.29 \pm 0.67$ & $2.01 \pm 0.67$ & $1.74 \pm 0.39$ & $1.65 \pm 0.32$ \\
\hline$t$ & \multirow{6}{*}{45} & & 4.127 & 5.169 & 3.594 & 3.419 \\
\hline$P$ & & & 0.033 & 0.012 & 0.017 & 0.022 \\
\hline \multirow{2}{*}{ Study group } & & Before treatment & $4.16 \pm 1.09$ & $4.33 \pm 1.22$ & $3.95 \pm 0.85$ & $3.71 \pm 0.79$ \\
\hline & & After treatment & $1.41 \pm 0.36^{\mathrm{a}}$ & $1.08 \pm 0.42^{\triangle}$ & $0.94 \pm 0.21^{\triangle}$ & $0.91 \pm 0.15^{\triangle}$ \\
\hline$t$ & & & 5.394 & 5.713 & 4.837 & 5.284 \\
\hline$P$ & & & 0.01 & 0.001 & 0.013 & 0.001 \\
\hline
\end{tabular}

The results were expressed as mean \pm standard deviation and analyzed by the $t$-test. ${ }^{\triangle}$ indicates $P<0.05$ compared with the control group.

TABLE 3: The classification of radiation-induced oral mucositis was evaluated in the control and observation group after treatment.

\begin{tabular}{lccccc}
\hline Group & Case & Grade 0 & Grade 1 & Grade 2 & Grade 3 \\
\hline Control group & 45 & $1(2.22 \%)$ & $14(31.11 \%)$ & $21(46.67 \%)$ & $7(15.56 \%)$ \\
Study group & 45 & $4(8.89 \%)$ & $18(40.00 \%)$ & $21(46.67 \%)$ & $2(4.44 \%)$ \\
$\chi^{2}$ & & & 2.203 & 0 \\
$P$ & & & 0.028 & \\
\hline
\end{tabular}

The results were described by ratio or percentage and analyzed by the chi-square test.

$\mathrm{CD}^{+4}$, and $\mathrm{CD}^{+8}(120 \mu \mathrm{L})$, all conjugated with fluorescin isothiocyanate (FITC) and analyzed by flow cytometry (FAC Scan, BD Pharmingen, U.S), and McAb of mouse anti-human IgG in positive control $(120 \mu \mathrm{L})$, which was examined by BIO450 enzyme-labelled instrument, and kept the solution away from light for 30 minutes. Centrifugation was performed again at the speed of $2000 \mathrm{R} / \mathrm{min}$ maintaining 15 minutes and rinsed with $2 \mathrm{~mL}$ PBS, and the above two procedures were performed one more time to get the supernatant but discarded it; finally, PBS $500 \mu \mathrm{L}$ was added into the solution. All data were analyzed by CellQuest Plot software.

2.7. Statistical Analysis. All data were processed by the SPSS 23.0 software. The measurement data were defined as mean \pm standard deviation and analyzed by the $t$-test. The counting data were described by ratio or percentage and analyzed by the chi-square test. A level of $P<0.05$ was considered statistically significance.

\section{Result}

3.1. Yunnan Baiyao plus Kangfuxin Solution Relieved the Symptoms of Irradiation-Induced Oral Mucositis. We first scored 90 NPC patients about their symptoms of oral mucositis including pain, mucosal hyperaemia and edema, mucosal ulceration, and dry mouth after radiotherapy. Each symptom was scored from 0 to 6 points. The lower the score was, the better the efficacy was. As shown in Table 2, although the scores of symptoms were all decreased after normal saline and inhalation of dexamethasone, gentamicin, and vitamin $B_{12}$ with or without additional treatment of Yunnan Baiyao plus Kangfuxin solution $(P<0.05)$, this decrease was greater for patients with additional treatment of Yunnan Baiyao plus Kangfuxin solution. The study group exhibited few points than the control group after treatment $(P<0.05)$. These data suggested that additional treatment of Yunnan Baiyao plus Kangfuxin solution relieved the symptoms of irradiation-induced oral mucositis in NPC patients after radiotherapy.

\subsection{Yunnan Baiyao plus Kangfuxin Solution Attenuated the} Disease Severity of Irradiation-Induced Oral Mucositis. Subsequently, the classification of radiation-induced oral mucositis was evaluated in the control and observation groups after normal saline and inhalation of dexamethasone, gentamicin, and vitamin $B_{12}$ with or without additional treatment of Yunnan Baiyao plus Kangfuxin solution. The control group had 1 case with no evidence of oral mucositis, 14 cases of grade 1, 21 cases of grade 2, 7 cases of grade 3, and 2 cases of grade 4 . The study group had 4 cases with no evidence of oral mucositis, 18 cases of grade 1, 21 cases of grade 2, 2 cases of grade 3 , and 0 case of grade 4 . It was revealed that the control group and the study group were significantly different with regard to occurrence of radiation-induced oral mucositis according to classifications $(P<0.05$, Table 3$)$.

3.3. Yunnan Baiyao plus Kangfuxin Solution Reduced Inflammatory Response in Irradiation-Induced Oral Mucositis. Early inflammation is a major factor of mucosal reactions to radiotherapy. Radiation-induced oral mucositis is associated with continuing presence of systemic inflammation. Given that, the release of inflammatory factors was examined by ELISA detection of serum CRP, TNF- $\alpha$, IL-1, and MMP-9 before and after normal saline and inhalation of dexamethasone, gentamicin, and vitamin $\mathrm{B}_{12}$ with or 

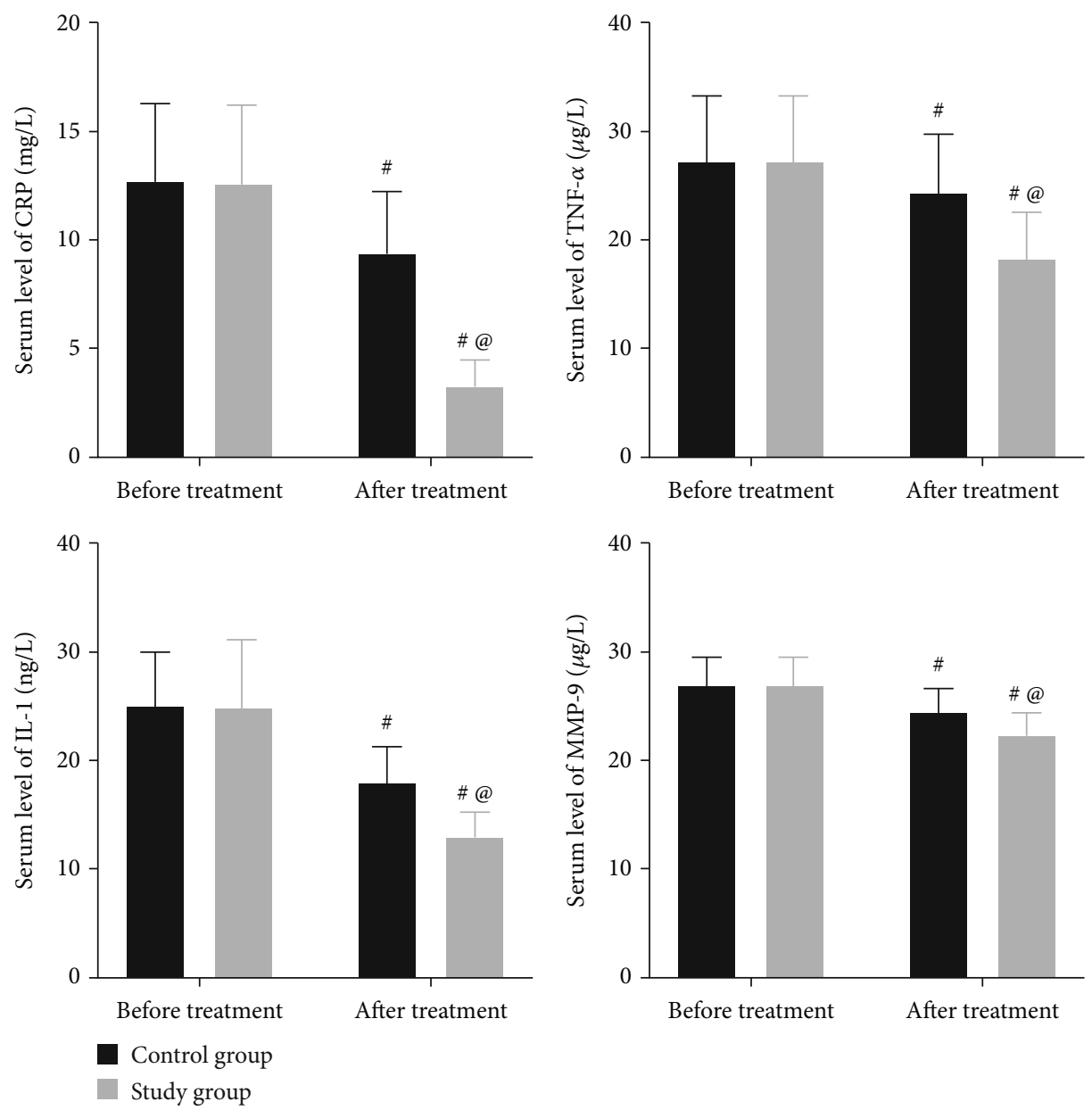

Figure 2: ELISA detection of serum levels of CRP, TNF- $\alpha$, IL-1, and MMP-9 in the control and study groups before and after treatment. The results were expressed as were defined as mean \pm standard deviation and analyzed by the $t$-test. \# indicates $P<0.05$ compared with before treatment, and @ indicates $P<0.05$ compared with the control group.

without additional treatment of Yunnan Baiyao plus Kangfuxin solution. Although the serum levels of CRP, TNF- $\alpha$, IL-1, and MMP-9 were all remarkably declined after normal saline and inhalation of dexamethasone, gentamicin, and vitamin $B_{12}$ with or without additional treatment of Yunnan Baiyao plus Kangfuxin solution $(P<0.05)$, the study group exhibited declined serum levels of CRP, TNF- $\alpha$, IL- 1 , and MMP-9 than the control group after treatment $(P<0.05$, Figure 2). These results indicated that additional treatment of Yunnan Baiyao plus Kangfuxin solution reduced inflammatory response in NPC patients with irradiation-induced oral mucositis.

3.4. Yunnan Baiyao plus Kangfuxin Solution Reduced HIF-1 $\alpha$ and VEGF Expressions in Irradiation-Induced Oral Mucositis. HIF- $1 \alpha$ is a transcription factor induced by hypoxia or inflammation and plays a pivotal role in physiological and pathological processes. Results of ELISA showed that the serum level of HIF- $1 \alpha$ in the two groups were significantly lower after treatment than those before treatment $(P<0.05)$, and the change was more significant in the study group than those in the control group $(P<0.05$, Figure 3$)$. VEGF is a potent angiogenic cytokine implicated in tumour vasculogen- esis. Previous evidence showed that VEGF was upregulated after ulceration. Our ELISA results revealed that the serum level of VEGF in the two groups were significantly lower after treatment than those before treatment $(P<0.05)$. The study group had a declined VEGF level when comparable to the control group $(P<0.05$, Figure 3$)$.

3.5. Yunnan Baiyao plus Kangfuxin Solution Sustained T Lymphocytes. Increasing evidence points that oral mucositis may be related with autoimmune dysregulation that is characterized by reduced $\mathrm{T}$ cells. The numbers of $\mathrm{CD}^{+3}, \mathrm{CD}^{+4} \mathrm{~T}$ cells and the ratio of $\mathrm{CD}^{+4} / \mathrm{CD}^{+8}$ were increased significantly, and the numbers of $\mathrm{CD}^{+8}$ were declined significantly after treatment than those before treatment $(P<0.05)$; these changes were more obvious in the study group compared with the control group $(P<0.05$, Table 4$)$.

\section{Discussion}

The management of oral mucositis mainly depends on local medication. Bacterial infection is often secondary to oral mucositis, which further reduces the tolerance of local normal tissues to radiation. Therefore, gentamicin is generally 

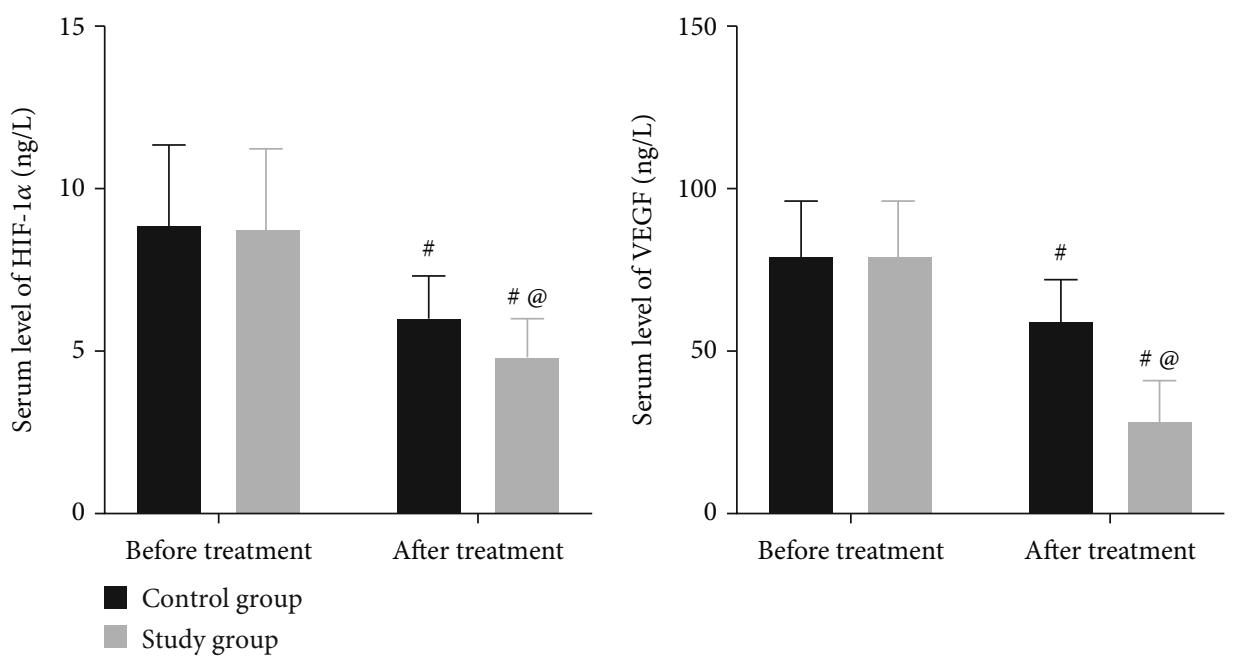

FIGURE 3: ELISA detection of serum levels of HIF- $1 \alpha$ and VEGF in the control and study group before and after treatment. The results were expressed as were defined as mean \pm standard deviation and analyzed by the $t$-test. \# indicates $P<0.05$ compared with before treatment, and @ indicates $P<0.05$ compared with the control group.

TABLE 4: The numbers of $\mathrm{CD}^{+3}, \mathrm{CD}^{+4}$, and $\mathrm{CD}^{+8}$ subpopulations of $\mathrm{T}$ cells and the ratio of $\mathrm{CD}^{+4} / \mathrm{CD}^{+8}$ in the control and study group before and after treatment.

\begin{tabular}{|c|c|c|c|c|c|c|}
\hline Group & Case & Time & $\mathrm{CD}^{+4}(\%)$ & $\mathrm{CD}^{+8}(\%)$ & $\mathrm{CD}^{+3}(\%)$ & $\mathrm{CD}^{+4} / \mathrm{CD}^{+8}$ \\
\hline \multirow{2}{*}{ Control group } & \multirow{2}{*}{45} & Before treatment & $36.56 \pm 10.52$ & $29.14 \pm 3.05$ & $54.33 \pm 12.85$ & $0.82 \pm 0.36$ \\
\hline & & After treatment & $40.29 \pm 10.43$ & $32.37 \pm 4.48$ & $69.94 \pm 12.57$ & $1.31 \pm 0.49$ \\
\hline$t$ & \multirow{4}{*}{45} & & 6.149 & 7.134 & 8.529 & 3.475 \\
\hline$P$ & & & 0.001 & 0.001 & 0.001 & 0.021 \\
\hline \multirow{2}{*}{ Study group } & & Before treatment & $36.46 \pm 10.03$ & $29.12 \pm 3.06$ & $53.91 \pm 12.87$ & $0.84 \pm 0.34$ \\
\hline & & After treatment & $48.14 \pm 11.04^{\mathrm{a}}$ & $37.19 \pm 6.10^{\triangle}$ & $80.65 \pm 10.99^{\triangle}$ & $1.73 \pm 0.65^{\triangle}$ \\
\hline$t$ & & & 7.021 & 10.102 & 24.395 & 4.839 \\
\hline$P$ & & & 0.001 & 0.001 & 0.001 & 0.016 \\
\hline
\end{tabular}

The results were expressed as mean \pm standard deviation and analyzed by the $t$-test. $\triangle$ indicates $P<0.05$ compared with the control group.

used in clinical antibacterial treatment for oral mucositis [15]. Dexamethasone can reduce the response of tissue to inflammatory factors and help to control inflammation in oral mucositis [16]. Vitamin $\mathrm{B}_{12}$ can promote the regeneration of epithelial cells, maintain the function of epithelial cells, and accelerate the healing of oral ulcer [17]. The patients in the control group were given an inhalation (15 min once, twice for one day) of 16 F.U gentamicin, $5 \mathrm{mg}$ dexamethasone, and $2 \mathrm{mg}$ vitamin $\mathrm{B}_{12}$.

Yunnan Baiyao is a traditional Chinese herbal medicine that has been used to treat wounds for over 100 years. It is called "holy medicine" for traumatology. It is good at promoting blood circulation to stop bleeding, dispersing blood stasis, and detumescence. It has the pharmacological effects of hemostasis, bacteriostasis, and removing blood stasis. It can promote the synthesis of collagen, promote the growth of epidermal cells, and improve the local microcirculation, which is conducive to the rapid repair of damaged mucosa [18].

Kangfuxin liquid is a drug extracted from the dried body of Fusarium falcatum, with effective components polyols and peptides [19]. It can promote fibroblasts to synthesize a large amount of collagen, which is conducive to the growth of epidermal cells in injured tissues, accelerate the proliferation of granulation tissue, induce the proliferation of mucosal capillary vessels, improve local blood supply, and facilitate the rapid repair of wounds [20,21]. Kangfuxin liquid contains mucosamine that can enhance the immune function and promote wound healing [22]. In this study, Yunnan Baiyao combined with Kangfuxin liquid was used in the prevention and treatment of oral mucositis after radiotherapy, and satisfactory results were achieved. The degree of oral mucositis of patients was milder, and the incidence of severe injury above grade 3 was significantly lower than that of patients who were treated with local application of normal saline combined with dexamethasone, gentamicin, and vitamin $B_{12}$ by aerosol inhalation. In addition, the safety of Yunnan Baiyao and Kangfuxin Liquid was good, and there was no special discomfort during the treatment.

The inflammation was induced in oral mucositis of patients. The level of CRP was increased in cancer patients after radiotherapy [23]. TNF- $\alpha$ is a cytokine secreted by the 
monocyte macrophage system, which has a variety of biological activities and participates in the occurrence and development of inflammatory diseases such as infection, trauma, autoimmune diseases, and malignant tumors [24]. IL-1, a member of the interleukin family, is produced by activated monocyte macrophages. It can stimulate T lymphocyte activation, synthesize CRP, and cause fever and cachexia [25]. TGF- $\beta 1$ is a member of the transforming growth factor family, which can transform the phenotype of normal fibroblasts [26]. MMP-9 is a member of matrix metalloproteinase family, which can degrade or remodel extracellular matrix and release TGF- $\beta 1$ [27]. In this study, we found that the levels of CRP, TNF- $\alpha$, IL- 1 , TGF- $\beta 1$, and MMP-9 in NPC patients with radiotherapy were significantly higher than those before radiotherapy. The results suggest that radiation injury can stimulate the body to produce inflammatory response, activate monocyte macrophages, and increase the levels of inflammatory factors. However, the levels of CRP, TNF- $\alpha$, IL-1, TGF- $\beta 1$, and MMP-9 in patients with adjuvant therapy of Yunnan Baiyao and Kangfuxin liquid were significantly reduced, suggesting that Yunnan Baiyao and Kangfuxin liquid can alleviate radiation-induced oral mucositis, which may be related to the fact that the glucosamine components in Kangfuxin liquid can regulate the synthesis of inflammatory substances such as interleukin, interferon, and leukotriene.

In summary, Yunnan Baiyao combined with Kangfuxin liquid effectively prevents radiotherapy-induced oral mucositis and reduces the release of inflammatory factors, compared with normal saline combined with dexamethasone, gentamicin, and vitamin $B_{12}$. Yunnan Baiyao combined with Kangfuxin liquid improves the quality of life in patients, and this approach is effective, user-friendly, safe, and appropriate for clinical application. Nevertheless, large-scale populations should be recruited to strengthen the validation of our results.

\section{Data Availability}

The data used to support the findings of this study are included within the article.

\section{Conflicts of Interest}

All authors declare that they have no conflict of interest.

\section{Authors' Contributions}

Xiuyu Tang and Jiahui Sun contributed equally to this work and regarded as co-first authors.

\section{References}

[1] Y. P. Chen, A. T. C. Chan, Q. T. Le, P. Blanchard, Y. Sun, and J. Ma, "Nasopharyngeal carcinoma," Lancet, vol. 394, no. 10192, pp. 64-80, 2019.

[2] R. Guo, Y. P. Mao, L. L. Tang, L. Chen, Y. Sun, and J. Ma, “The evolution of nasopharyngeal carcinoma staging," The British Journal of Radiology, vol. 92, no. 1102, p. 20190244, 2019.
[3] X. S. Sun, X. Y. Li, Q. Y. Chen, L. Q. Tang, and H. Q. Mai, "Future of Radiotherapy in Nasopharyngeal Carcinoma," The British Journal of Radiology, vol. 92, no. 1102, p. 20190209, 2019.

[4] A. W. M. Lee, W. T. Ng, J. Y. W. Chan et al., "Management of locally recurrent nasopharyngeal carcinoma," Cancer Treatment Reviews, vol. 79, p. 101890, 2019.

[5] P. J. Li, K. X. Li, T. Jin et al., "Predictive model and precaution for oral mucositis during chemo-radiotherapy in nasopharyngeal carcinoma patients," Frontiers in Oncology, vol. 10, p. 596822, 2020.

[6] X. X. Zhu, X. J. Yang, Y. L. Chao et al., "The potential effect of oral microbiota in the prediction of mucositis during radiotherapy for nasopharyngeal carcinoma," eBioMedicine, vol. 18, pp. 23-31, 2017.

[7] M. Agulnik and J. B. Epstein, "Nasopharyngeal carcinoma: current management, future directions and dental implications," Oral Oncology, vol. 44, no. 7, pp. 617-627, 2008.

[8] R. V. Lalla, D. P. Saunders, and D. E. Peterson, "Chemotherapy or radiation-induced oral mucositis," Dental Clinics of North America, vol. 58, no. 2, pp. 341-349, 2014.

[9] M. Kishimoto, M. Akashi, K. Tsuji et al., "Intensity and duration of neutropenia relates to the development of oral mucositis but not odontogenic infection during chemotherapy for hematological malignancy," PLOS ONE, vol. 12, no. 7, p. e0182021, 2017.

[10] W. Parulekar, R. Mackenzie, G. Bjarnason, and R. C. Jordan, "Scoring oral mucositis," Oral Oncology, vol. 34, no. 1, pp. 63-71, 1998.

[11] J. L. Ren, H. Dong, Y. Han et al., "Network pharmacology combined with metabolomics approach to investigate the protective role and detoxification mechanism of _Yunnan Baiyao_formulation," Phytomedicine, vol. 77, p. 153266, 2020.

[12] X. Ren, Y. Zhu, L. Xie, M. Zhang, L. Gao, and H. He, "Yunnan Baiyao diminishes lipopolysaccharide-induced inflammation in osteoclasts," Journal of Food Biochemistry, vol. 44, no. 6, p. e13182, 2020.

[13] Y. Luo, M. Feng, Z. Fan et al., "Effect ofKangfuxinSolution on Chemo/Radiotherapy-Induced Mucositis in Nasopharyngeal Carcinoma Patients: A Multicenter, Prospective Randomized Phase III Clinical Study," Evidence-Based Complementary and Alternative Medicine, vol. 2016, Article ID 8692343, 7 pages, 2016.

[14] J. Bardy, A. Molassiotis, W. D. Ryder et al., "A double-blind, placebo-controlled, randomised trial of active manuka honey and standard oral care for radiation-induced oral mucositis," The British Journal of Oral \& Maxillofacial Surgery, vol. 50, no. 3, pp. 221-226, 2012.

[15] D. P. Saunders, On behalf of the Mucositis Study Group of the Multinational Association of Supportive Care in Cancer/ International Society of Oral Oncology (MASCC/ISOO), T. Rouleau et al., "Systematic review of antimicrobials, mucosal coating agents, anesthetics, and analgesics for the management of oral mucositis in cancer patients and clinical practice guidelines," Support Care Cancer, vol. 28, no. 5, pp. 24732484, 2020.

[16] P. Molina Prats, F. Gomez Garcia, F. Martinez Diaz, R. Amaral Mendes, and P. Lopez-Jornet, "The therapeutic effects of apigenin and dexamethasone on 5-fluorouracil-induced oral mucositis - a pilot study using a Syrian hamster model," Journal of Oral Pathology \& Medicine, vol. 46, no. 2, pp. 142-147, 2017. 
[17] G. Liang, W. Du, Q. Ke, B. Huang, and J. Yang, "The effects of recombinant human granulocyte colony-stimulating factor mouthwash on radiotherapy-induced oral mucositis in locally advanced nasopharyngeal carcinoma patients," Advances in Clinical and Experimental Medicine, vol. 26, no. 3, pp. 409413, 2017.

[18] S. C. Lenaghan, L. Xia, and M. Zhang, "Identification of nanofibers in the Chinese herbal medicine: Yunnan Baiyao," Journal of Biomedical Nanotechnology, vol. 5, no. 5, pp. 472-476, 2009.

[19] K. S. Qu, Y. Li, Y. Liang et al., "KangFuXin Liquid in the Treatment of Diabetic Foot Ulcer: A Systematic Review and MetaAnalysis," Evidence-Based Complementary and Alternative Medicine, vol. 2019, Article ID 3678714, 10 pages, 2019.

[20] J. B. Zou, X. F. Zhang, Y. J. Shi et al., "Therapeutic Efficacy of Kangfuxin Liquid Combined with PPIs in Gastric Ulcer," Evidence-Based Complementary and Alternative Medicine, vol. 2019, Article ID 1324969, 13 pages, 2019.

[21] X. J. Ma and G. Y. Huang, "Current status of screening, diagnosis, and treatment of neonatal congenital heart disease in China," World Journal of Pediatrics, vol. 14, no. 4, pp. 313314, 2018.

[22] H. Yao, S. Wei, Y. Xiang et al., "Kangfuxin Oral Liquid Attenuates Bleomycin-Induced Pulmonary Fibrosis via the TGF- $\beta 1 /$ Smad Pathway," Evidence-Based Complementary and Alternative Medicine, vol. 2019, Article ID 5124026, 14 pages, 2019.

[23] E. M. Thurner, S. Krenn-Pilko, U. Langsenlehner et al., "The elevated C-reactive protein level is associated with poor prognosis in prostate cancer patients treated with radiotherapy," European Journal of Cancer, vol. 51, no. 5, pp. 610-619, 2015.

[24] J. R. Bradley, "TNF-mediated inflammatory disease," The Journal of Pathology, vol. 214, no. 2, pp. 149-160, 2008.

[25] C. A. Dinarello, "Interleukin-1 in the pathogenesis and treatment of inflammatory diseases," Blood, vol. 117, no. 14, pp. 3720-3732, 2011.

[26] K. K. Kim, D. Sheppard, and H. A. Chapman, "TGF- $\beta 1$ Signaling and Tissue Fibrosis," Cold Spring Harbor Perspectives in Biology, vol. 10, no. 4, p. a022293, 2018.

[27] H. Huang, "Matrix Metalloproteinase-9 (MMP-9) as a Cancer Biomarker and MMP-9 Biosensors: Recent Advances," Sensors, vol. 18, no. 10, p. 3249, 2018. 